\title{
COMPORTAMENTO DE HIBRIDOS DE CEBOLA NAS CONDIÇÕES DE MONTE ALEGRE DO SUL, SP (1)
}

\author{
ROGÉRIO SALLES LISBÃO $(2,5)$, JOÃO BAPTISTA FORNASIER $(2,5)$, \\ TOSHIO IGUE $(3,5)$ e RUI RIBEIRO DOS SANTOS $\left({ }^{4}\right)$
}

\begin{abstract}
RESUMO
Avaliou-se o comportamento de cinco híbridos comerciais de cebola (Allium cepa L.) - Granex 33, Granex 429, Baia Ouro AG-55, Baia Ouro AG-55R e Baia Ouro AG-59 e cinco experimentais - FMX-151, FMX-179, FMX-181, FMX-182 e FMX-183 quanto à qualidade e produtividade, visando introduzi-los e indicar aos produtores os melhores para o cultivo nas condiçôes de Monte Alegre do Sul, SP, e áreas de ecologia similar. O experimento foi conduzido na Estação Experimental do Instituto Agronômico, situada naquela localidade, de 21 de março (semeadura) a 7 de novembro de 1983 (última colheita). Nas suas condiçôes, outono-inverno, verificou-se que quanto à produtividade de bulbos comerciáveis, os híbridos Granex 33 (testemunha), Baia Ouro AG-55 e Baia Ouro AG-59 foram superiores aos híbridos FMX-181 e FMX-182, não diferindo de Baia Ouro AG-55R, Granex 429, FMX-151, FMX-179 e FMX-183. Em relação ao peso médio de bulbos comerciáveis, os de melhor comportamento foram Baia Ouro AG-55 e Baia Ouro AG-59, que superaram Baia Ouro AG-55R, Granex 429, FMX-181 e FMX-182, porém não diferiram de Granex 33, FMX-151,
\end{abstract}

(1) Apresentado no XXV Congresso Brasileiro de Olericultura, em Blumenau, SC, em 15-19 de julho de 1985. Recebido para publicação em 26 de junho de 1985.

(2) Seção de Hortaliças Diversas, Instituto Agronômico (IAC), Caixa Postal 28, 13001 Campinas (SP).

(3) Seção de Técnica Experimental e Cálculo, IAC.

$\left({ }^{4}\right)$ Estaçăo Experimental de Monte Alegre do Sul, IAC.

(5) Com bolsa de suplementação do CNPq. 
FMX-179 e FMX-183. O hibrido Baia Ouro AG-55R e os experimentais FMX-181, FMX-182 e FMX-183 apresentaram porcentagens mais elevadas de bulbos tipo "charuto", tendo os três últimos, ainda, maior desuniformidade quanto ao formato de bulbos. Não se verificou ocorrência de florescimento prematuro em nenhum material avaliado. Quanto ao ciclo, o mais precoce foi Granex 33 e o mais tardio, Baia Ouro AG-55. Em relação às características consideradas, •podem-se indicar os hîbridos Granex 33, Granex 429, Baia Ouro AG-55, Baia Ouro AG-59, FMX-151 e FMX-179, como de aptidão ao cultivo em regiōes de ecologia similar à de Monte Alegre do Sul.

Termos de indexação: cebola (Allium cepa L.), híbridos, comportamento.

\section{INTRODUÇÃO}

A cebola (Allium cepa L), hortaliça de grande expressão econômica no País e no Estado de São Paulo, participou com $35 \%$ da produção nacional em 1983 (ANUÁRIO ESTATISTICO DO BRASIL, 1983). A procura de híbridos promissores, pelos produtores dessa hortaliça em Monte Alegre do Sul, SP, tem aumentado ultimamente, e dentre as características preferenciais são referidas, como mais relevantes, a precocidade de ciclo, a uniformidade dos bulbos e a alta produtividade.

Nas condiçōes climáticas do Estado de São Paulo, são poucos os trabalhos objetivando pesquisas de comportamento de novos híbridos com aptidão comercial.

CHURATA-MASCA \& SAITO (1979), estudando o comportamento de hibridos comerciais e experimentais de cebola de várias origens, constataram, na região de Monte Alto, SP, maior produtividade e porcentagem de bulbos graúdos nos híbridos Granex 33 e Granex 3178. CHURATA-MASCA \& SANTOS (1983) obtiveram, ainda nas condiçōes de Monte Alto, maior produtividade com os híbridos Granex Yellow, Granex 33, Granex 429, Baia Ouro AG-59, e com as variedades Excel e IPA-2.

LEITE JR. et alii (1984) constataram em Jaboticabal, SP, produção total e comerciável na variedade Texas Grano 502, em confronto com outros cultivares e o hibrido Granex 33.

A cebola é uma hortaliça cuja adaptação em deteminada localidade é condicionada por fatores ambientes, notadamente o fotoperiodo e a temperatura (JONES \& MANN, 1963). Dessa maneira, se as condições climáticas não satisfizerem às exigências próprias do material em cultivo, pode ocorrer a não-bulbificação, a emissão precoce do pendão floral, a formação de "charutos" e de pequenos bulbos (MASCARENHAS, 1980). Em vista disso, da importância econô- 
mica da cebola e da procura de hibridos promissores em Monte Alegre do Sul e regiōes produtoras de ecologia similar, o presente experimento pretende indicar os melhores materiais quanto à produtividade e qualidade do bulbo produzido, bem como avaliar a sua adaptabilidade e extrair outras informaçōes a respeito dos híbridos em fase de testes de aptidão comercial.

\section{MATERIAL E MÉTODOS}

Estudou-se o comportamento de cinco híbridos comerciais de cebola e cinco experimentais, de diferentes origens, selecionados para produzir em condiçóes de dias curtos. Os híbridos comerciais estudados foram os seguintes: Granex 33, Granex 429, Baia Ouro AG-55, Baia Ouro AG-55R e Baia Ouro AG-59. Os dois primeiros foram desenvolvidos pela companhia de sementes Asgrow, nos EUA, e os três últimos pela Agroceres S.A., no Brasil. Destes, o Baia Ouro AG$55 R$ é um hibrido reciproco do Baia Ouro AG-55. Os referidos híbridos vêm sendo utilizados pelos plantadores de cebola no Estado de São Paulo, sendo que o Granex 33 e o Granex 429 são conhecidos há alguns anos.

Os híbridos experimentais FMX-151, FMX-179, FMX-181, FMX-182 e FMX-183, por sua vez, foram obtidos pela firma de sementes Ferry Morse, nos EUA. Contudo, é desconhecido seu comportamento nas condiçōes edafoclimáticas paulistas.

A cebola Granex 33 foi escolhida como testemunha, em razão de seu uso na cultura, e de ser a mais adaptada à latitude do Estado de São Paulo, nas condições de cultivo de outono-inverno.

O experimento foi instalado na Estação Experimental do Instituto Agronômico em Monte Alegre do Sul - SP, localizada a 777 metros de altitude, $22^{\circ} 42^{\circ}$ de latitude sul e $46^{\circ} 39^{\prime}$ de longitude oeste, em solo tipo aluvião, unidade Moranguinho, de textura fino-areno-barrenta, segundo ROTTA et alii (1971). A análise química do solo, efetuada pela Seção de Fertilidade do Solo e Nutrição de Plantas, do Instituto Agronômico, revelou os seguintes resultados: $\mathrm{pH}$ em $\mathrm{CaCl}_{2}-4,8$, $\mathrm{MO}-2,2 \%, \mathrm{P}$ resina $-164 \mu \mathrm{g} / \mathrm{cm}^{3}, \mathrm{~K}-0,21, \mathrm{Ca}-2,2, \mathrm{Mg}-0,5, \mathrm{H}+\mathrm{Al}-4,3, \mathrm{~S}$ $-2,9$ e T $-7,2 \mathrm{meq} / 100 \mathrm{~cm}^{3}$ e saturação em bases $40 \%$.

Considerando esses resultados, realizou-se a calagem com 60 dias de antecedência ao transplante das mudas, incorporando-se $300 \mathrm{~g} / \mathrm{m}^{2}$ de calcário dolomítico. A adubação de plantio, por sua vez, constou da aplicação, por metro quadrado, de $1,0 \mathrm{~kg}$ de matéria orgânica, $50 \mathrm{~g}$ de superfosfato simples e $15 \mathrm{~g}$ de cloreto de potássio. Em cobertura, aplicou-se o sulfato de amônio, em duas parcelas iguais de $15 \mathrm{~g}$ por metro quadrado, aos 30 e 45 dias após o transplante das mudas. 
A cultura foi conduzida pelo método de plantio com mudas. Assim, a semeadura foi efetuada em 21 de março de 1983, o transplante das mudas em 20 de maio de 1983 e as colheitas, de 21 de setembro a 7 de novembro de 1983. As práticas culturais foram efetuadas nos moldes nomais recomendados para a cultura (CAMPOS, 1969). A irrigação foi suspensa cerca de 15 dias antes de cada colheita, efetuada por ocasião da seca e do tombamento das ramas. As temperaturas médias mensais do ar foram de $21,1,20,3,19,1,17,2,17,6,17,2$, $17,6,20,0$ e $21,2^{\circ} \mathrm{C}$, respectivamente, para os meses de março (semeadura) a novembro (última colheita). A temperatura mínima registrada foi de $4,3^{\circ} \mathrm{C}$, em agosto, e a máxima, de $33,2^{\circ} \mathrm{C}$, em novembro. A média das minimas, para os nove meses, foi de $14,8^{\circ} \mathrm{C}$, e a das máximas, de $25,6^{\circ} \mathrm{C}\left({ }^{6}\right)$.

Empregou-se o delineamento de blocos ao acaso, com dez tratamentos e quatro repetiçōes. Cada parcela, com uma área de $4,08 \mathrm{~m}^{2}$, era constituilda de seis linhas de 17 plantas, espaçadas de $0,40 \mathrm{~m} \times 0,10 \mathrm{~m}$. Para a avaliação da produtividade e de outras caracteristicas, foram consideradas as quatro linhas internas de cada canteiro, no total de 60 plantas úteis, ocupando uma área de $2,40 \mathrm{~m}^{2}$.

As plantas colhidas foram curadas à sombra por um perfodo de 25 dias, sendo em seguida os bulbos desprovidos das ramas e classificados em comerciấveis: de primeira, segunda e terceira, e não-comerciåveis: consenva, múltiplos (bulbos defeituosos unidos pela base) e "charutos" (cebolas compridas, lembrando o alho-porro). Os bulbos comerciáveis foram classificados de acordo com o diâmetro transversal em: de primeira (diâmetro $>55 \mathrm{~mm}$ ), de segunda (diâmetro entre 40 e $55 \mathrm{~mm}$ ) e de terceira (diâmetro entre 25 e $40 \mathrm{~mm}$ ), conforme CAMPOS (1969). Após a classificação, os bulbos comerciåveis foram contados e pesados, sendo os resultados expressos em porcentagem e quilograma por hectare respectivamente. Os bulbos não-comerciáveis foram apenas contados, sendo os resultados expressos em porcentagem. Determinaram-se, também, o fndice de sobrevivência das plantas (número de plantas colhidas em relação ao número plantado) e o ciclo da cultura, com os dados expressos em porcentagem e dias respectivamente.

\section{RESULTADOS E DISCUSSÃO}

Os dados de produtividade e peso médio dos bulbos comerciáveis (tipos de primeira, segunda e terceira), dos dez híbridos de cebola estudados, encontram-se no quadro 1.

A análise estatística mostrou diferenças significativas, pelo teste $F$, entre os híbridos avaliados, quanto à produtividade e peso médio de bulbos comerciáveis. Em relação ao peso médio dos bulbos, a diferença foi altamente significativa.

(6) Dados fornecidaś peia Seção de Climatologia Agrf́cola, Instituto Agronômico. 
QUADRO 1. Produtividade e peso médio de bulbos comerciáveis, obtidos no ensaio de comportamento de híbridos de cebola (Allium cepa L.), realizado na Estação Experimental de Monte Alegre do Sul, SP ( $\left.{ }^{1}\right)$

\begin{tabular}{lcc}
\hline & \multicolumn{2}{c}{ Bulbos comerciáveis } \\
\cline { 2 - 3 } & Produtividade & Peso médio \\
\hline & $\mathrm{kg} / \mathrm{ha}$ & $\mathrm{g}$ \\
Granex 33 & $\left.22.708 \mathrm{a}^{2}\right)$ & $97,21 \mathrm{abcd}\left(^{2}\right)$ \\
Baia Ouro AG-59 & $22.511 \mathrm{a}$ & $114,10 \mathrm{a}$ \\
Baia Ouro AG-55 & $21.677 \mathrm{a}$ & $114,66 \mathrm{a}$ \\
FMX-151 & $19.292 \mathrm{ab}$ & $107,73 \mathrm{ab}$ \\
Baia Ouro AG-55R & $18.938 \mathrm{ab}$ & $88,00 \mathrm{bcaje}$ \\
FMX-179 & $18.833 \mathrm{ab}$ & $104,13 \mathrm{abc}$ \\
Granex 429 & $16.406 \mathrm{ab}$ & $81,71 \mathrm{de}$ \\
FMX-183 & $15.458 \mathrm{ab}$ & $94,44 \mathrm{abcde}$ \\
FMX-181 & $13.667 \mathrm{~b}$ & $79,66 \mathrm{e}$ \\
FMX-182 & $12.865 \mathrm{~b}$ & $83,57 \mathrm{cde}$ \\
\hline F & $2,32^{*}$ & $3,72^{* *}$ \\
CV (\%) & 25,48 & 14,18 \\
\hline
\end{tabular}

(1) Média de quatro repetiçōes.

(2) Letras não comuns indicam diferenças significativas pelo teste de Duncan a $5 \%$.

A produtividade obtida para os híbridos estudados variou de 12.865 a $22.708 \mathrm{~kg} / \mathrm{ha}$, sendo que o Granex 33 (testemunha), Baia Ouro AG-59 e Baia Ouro AG-55 apresentaram valores médios superiores a $20.000 \mathrm{~kg} / \mathrm{ha}$, resultados considerados elevados em relação ao rendimento médio paulista $(14.975 \mathrm{~kg} / \mathrm{ha})$, estimado em 1983 (ANUÁRIO ESTATISTICO DO BRASIL, 1983). Por outro lado, a comparação das médias de produtividade, através do teste de Duncan, ao nível de $5 \%$, mostrou que esses três hibridos comerciais foram estatisticamente superiores apenas aos hibridos experimentais $F M X-181$ e FMX-182, nāo diferindo, portanto, de FMX-151, Baja Ouro AG-55R, FMX-179, Granex 429 e FMX-183. O alto rendimento obtido pelo híbrido Granex 33 concorda com os dados encontrados por CHURATA-MASCA \& SAITO (1979) e CHURATA-MASCA \& SANTOS (1983), nas condições climáticas de Monte Alto, porém não com os conseguidos por LElTE JR. et alii (1984), em Jaboticabal. O bom comportamento do híbrido Baia Ouro AG-59, quanto à produtividade, foi também concorde com o resultado obtido por CHURATA-MASCA \& SANTOS (1983). 
O peso médio dos bulbos variou de 79,66 a 114,66g, aos 25 dias de colheita. A comparação das médias obtidas para esta característica, pelo teste de Duncan, ao nivel de 5\%, mostrou que os híbridos Baia Ouro Ag-55 e Baia Ouro AG-59 foram estatisticamente superiores aos híbridos Baia Ouro AG-55R, FMX-182, Granex 429 e FMX-181, mas não diferiram dos híbridos FMX-151, FMX-179, Granex 33 e FMX-183. De maneira geral, no espaçamento utilizado, $0,40 \times 0,10 \mathrm{~m}$, os bulbos atingiram peso adequado para a comercialização. Nas condições deste experimento, não foram observados bulbos com peso acima de $150 \mathrm{~g}$, os quais apresentam problemas de comercialização.

O híbrido Baia Ouro AG-55R, recíproco do Baia Ouro AG-55, apresentou, quanto ao peso médio de bulbos, comportamento diferente. Este fato sugere que os progenitores desses materiais não sejam geneticamente semelhantes.

Finalmente, pode-se verificar que apenas os híbridos experimentais FMX-181 e FMX-182, quanto à produtividade, e FMX-181, quanto ao peso médio de bulbos, apresentaram comportamentos inferiores ao híbrido Granex 33, utilizado como testemunha.

No quadro 2 são apresentados os resultados da classificação dos bulbos em comerciáveis (primeira, segunda e terceira) e não-comerciáveis (tipo conserva, múltiplos, pendoados e "charuto"), bem como o indice de sobrevivência das plantas e ciclo da cultura. Esses dados mostram que quanto à porcentagem de bulbos comerciáveis, o híbrido Granex 33 (testemunha) e o híbrido experimental FMX-179 apresentaram os maiores valores de bulbos tipo primeira (diâmetro transversal maior que $55 \mathrm{~mm}$ ). Quanto à formação de bulbos tipo segunda (diâmetro transversal entre 40 e $55 \mathrm{~mm}$ ), sobressafram-se os hifbridos comerciais Baia Ouro AG-55R, Granex 33, Baia Ouro AG-55, Baia Ouro AG-59 e Granex 429, com frequêencias superiores a $30 \%$. Os hibridos experimentais FMX-181 e FMX-182 apresentaram os menores valores totais de bulbos comerciáveis, com freqüência inferior a $70 \%$.

Quanto à formaçāo de bulbos tipo conserva (diâmetro menor que $25 \mathrm{~mm}$ ), as maiores porcentagens foram obtidas pelos híbridos experimentais FMX-151, FMX-179, FMX-181, FMX-182 e FMX-183, bem como pelos comerciais Baia Ouro AG-55 e Baia Ouro AG-59.

A freqũência de bulbos tipo "charuto", por sua vez, foi maior (acima de 10\%) nos híbridos FMX-181, FMX-182, FMX-183 e Baia Ouro AG-55R.

Em relação å formação de bulbos múltiplos, observaram-se valores inferiores a 2,0\% nos cinco hibridos experimentais: FMX-151, FMX-179, FMX-181, FMX-182 e FMX-183.

A produção sensivelmente maior ou menor de bulbos tipo "charuto" e bulbos múltiplos parece indicar diferenças de adaptabilidade dos materiais estudados às condiçōes climåticas locais (JONES \& MANN, 1963; MASCARENHAS, 1980). 


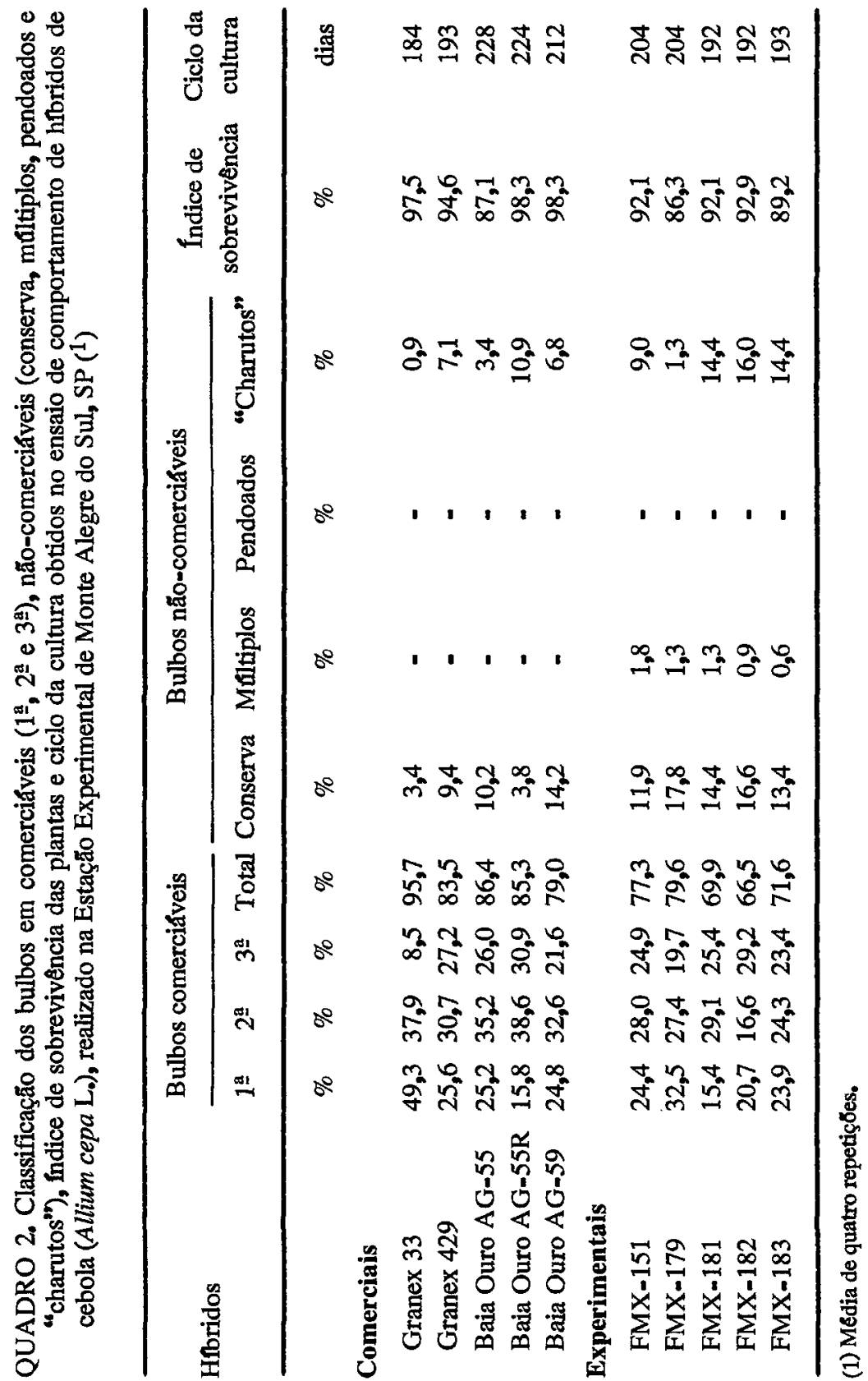


Não se verificou ocorrência de florescimento prematuro em nenhum dos híbridos estudados. Esse fato pode estar relacionado à não-ocorrência de temperaturas frias indutivas do pendoamento (THOMPSON \& SMITH, 1938), bem como à tolerância desses híbridos à referida característica.

Observourse um prolongamento no ciclo dos híbridos, o qual variou de 184 (Granex 33, mais precoce) a 228 dias (Baia Ouro AG-55, mais tardio), sendo superior ao esperado, provavelmente devido à ocorrência de precipitações elevadas e temperaturas mais frias, na época de formação e maturação dos bulbos.

Quanto à porcentagem de sobrevivência de plantas, os indices encontrados foram excelentes: todos os híbridos apresentaram valores superiores a $86 \%$.

A ocorrência de mancha-púrpura (Alternaria porri (Ell.) Cit.) foi maior no Granex 33 (testemunha) e no Granex 429, e menor nos híbridos Baia Ouro AG-55, Baia Ouro AG-55R e Baia Ouro AG-59. Quanto aos híbridos experimentais, a incidência da doença ocorreu em niveis intermediários.

Os hibridos experimentais FMX-181, FMX-182 e FMX-183 apresentaram maior desuniformidade no formato dos bulbos, enquanto os mais unifomes foram: Granex 33, Granex 429, Baia Ouro AG-55, Baia Ouro AG-55R e FMX-151. O FMX-179 e o Baia Ouro AG-59 tiveram unifomidade apenas regular. Em relação ao diåmetro da haste (coleto) e coloração da casca, todos os híbridos apresentaram bom aspecto.

\section{CONCLUSÃO}

Os resultados obtidos nas condições experimentais de Monte Alegre do Sul, para o perfodo outono-inverno, em 1983, em relação ao conjunto de caracteństicas agronômicas (produtividade, peso médio de bulbos, indice de sobrevivência das plantas, ciclo, características da planta, formato do bulbo, coloração da casca do bulbo, e freqũência de bulbos comerciåveis e não-comerciáveis) possibilitam indicar o cultivo, nas condições da referida localidade e de ecologia similar, dos híbridos comerciais Granex 33, Granex 429, Baia Ouro AG-55 e Baia Ouro AG59 , e aqueles em testes experimentais FMX-151 e FMX-179.

\section{SUMMARY}

BEHAVIOR OF ONION (ALLIUM CEPA L.) HYBRIDS, AT MONTE ALEGRE DO SUL, STATE OF SÃO PAULO, BRAZIL

A study was carried out at the Monte Alegre do Sul Experiment Station, State of São Paulo, Brazil ( $\left.22^{\circ} 42^{\prime} \mathrm{S}\right)$, to evaluate five short day 
marketable onion (Allium cepa L.) hybrids (Granex 33, Granex 429, Baia Ouro AG-55, Baia Ouro AG-55R and Baia Ouro AG-59) and five short day experimental onion hybrids (FMX-151, FMX-179, FMX-181, FMX-182 and FMX-183) from different origins. The sowing date was March 21, 1983 and the harvest period occurred from September 21 to November 7. The hybrids Granex 33, Baia Ouro AG-55 and Baia Ouro AG-59 yielded more than the hybrids FMX-181 and FMX-182. However, their yields were not statistically different from those of the following hybrids: Baia Ouro AG-55R, Granex 429, FMX-151, FMX-179 and FMX-183. In relation to the average weight of marketable bulbs, Baia Ouro AG-55 and Baia Ouro AG-59, were statistically superior to Baia Ouro AG-55R, Granex 429, FMX-181, and FMX-182. Considering type and shape of bulbs, Baia Ouro AG-55R, FMX-181, FMX-182 and FMX-183 showed higher porcentages of "charuto" type of bulbs than the others, while FMX-181, FMX-182 and FMX-183 presented also higher desuniformity in bulb shapes. The occurrence of "bolting" was not observed. In relation to the vegetative cycle, Granex 33 was the earliest while Baia Ouro AG-55 was the latest. The onion hybrids Granex 33, Granex 429, Baia Ouro AG-55, Baia Ouro AG-59, FMX-151 and FMX-179 were recommended to the growers of Monte Alegre do Sul, and areas of similar climatic conditions.

Index terms: onion (Allium cepa L.), hybrids, behavior.

\section{REFERÊNCIAS BIBLIOGRÁFICAS}

ANUÁRIO ESTATÍSTICO DO BRASIL. Rio de Janeiro, IBGE, v.44, 1983.

CAMPOS, H.R. de. Instruçōes para a cultura da cebola. 2.ed. Campinas, Instituto Agronômico, 1969. (Boletim, 164)

CHURATA-MASCA, M.G.C. \& SAITO, S.Y. Competição de cultivares híbridas de cebola na região de Monte Alto - SP. In: CONGRESSO BRASILEIRO DE OLERICULTURA, 19., Florianópolis, 1979. Resumos. Florianópolis, EMPASC,1979. v.1, p.40-41.

\& SANTOS, M.A.P. dos, Competição de cultivares de cebola.I - Monte AltoSP. In: CONGRESSO BRASILEIRO DE OLERICULTURA, 23., Rio de Janeiro, 1983. Resumos. Rio de Janeiro, 1983. p.36.

JONES, H.A. \& MANN, L.K. Onions and their allies: botany, cultivation and utilization. New York, Interscience, 1963. 286p.

LEITE JÚNIOR, A.P.; ATHAYDE, M.L.F. \& CHURATA-MASCA, M.G.C. Competição de cultivares de cebola em Jaboticabal, SP. In: CONGRESSO BRASILEIRO DE OLERICULTURA, 24., e REUNIĀO LATINO-AMERICANA DE OLERICULTURA, 1., Jaboticabal, 1984. Resumos. Jaboticabal, 1984. p.24.

MASCARENHAS, M.H.T. Cultivares de cebola. Informe Agropecuário, Belo Horizonte, 6(62):17-20, 1980.

ROTTA, C.L.; JORGE, J.A.; OLIVEIRA, J.B. de \& KÜPPER, A. Levantamento pedol6gico detalhado da Estaçăo Experimental de Monte Alegre do Sul, SP. Bragantia, Campinas, 30:215-276, 1971.

THOMPSON, H.C. \& SMITH, O. Seedstalk and bulb development in the onion (Allium cepa L.) Cornell Agric. Exp. Sta., 1938. 21p. (Bulletin, 708) 